\title{
Biomarkers associated with bronchopulmonary dysplasia/mortality in premature infants
}

\author{
Jessica Balena-Borneman' ${ }^{1}$ Namasivayam Ambalavanan², Hemant K. Tiwari', Russell L. Griffin ${ }^{3}$, Brian Halloran² and \\ David Askenazi
}

BACKGROUND: Bronchopulmonary dysplasia (BPD) portends lifelong organ impairment and death. Our ability to predict BPD in first days of life is limited, but could be enhanced using novel biomarkers.

METHODS: Using an available clinical and urine biomarker database obtained from a prospective 113 infant cohort (birth weight $\leq 1,200 \mathrm{~g}$ and/or gestational age $\leq 31 \mathrm{wk}$ ), we evaluated the independent association of 14 urine biomarkers with BPD/ mortality.

RESULTS: Two of the 14 urine biomarkers were independently associated with BPD/mortality after controlling for gestational age (GA), small for gestational age (SGA), and intubation status. The best performing protein was clusterin, a ubiquitously expressed protein and potential sensor of oxidative stress associated with lung function in asthma patients. When modeling for BPD/mortality, the independent odds ratio for maximum adjusted urine clusterin was 9.2 (95\% Cl: 3.3-32.8, $P<0.0001$ ). In this model, clinical variables (GA, intubation status, and SGA) explained $38.3 \%$ of variance; clusterin explained an additional 9.2\%, while albumin explained an additional 3.4\%. The area under the curve incorporating clinical factors and biomarkers was 0.941 .

CONCLUSION: Urine clusterin and albumin may improve our ability to predict BPD/mortality. Future studies are needed to validate these findings and determine their clinical usefulness.

$E^{*}$ xtremely premature infants are at high risk of bronchopulmonary dysplasia (BPD), which is characterized by inhibition of lung development and varying degrees of lung inflammation, fibrosis, and abnormal vascular remodeling $(1,2)$. Infants with BPD are at risk of lifelong impairment in pulmonary function, and death within the first few years of life (3). Infants are currently diagnosed with BPD based on their need for oxygen support at $36 \mathrm{wk}$ postmenstrual age (PMA) (4). Since death is a competing outcome for BPD, BPD/mortality at $36 \mathrm{wk}$ PMA is commonly used as an important shortterm outcome in extremely preterm infants.
Currently, it is difficult to determine which infants will develop BPD early in the disease course. Reliable biomarkers could help identify premature infants at risk of developing $\mathrm{BPD}$, and eventually may aid in earlier implementation of preventive measures. We have recently shown that acute kidney injury (AKI) is associated with development of BPD/death in preterm infants (5). We have also shown in previous studies that urinary biomarkers are associated with AKI in preterm infants (6), and that urinary biomarkers vary by gestational age and postnatal age (7).

We used the convenient sample of 14 urine proteins and clinical data from a prospective cohort of 113 premature infants (birth weight $\leq 1,200 \mathrm{~g}$ and/or gestational age $\leq 31 \mathrm{wk}$ ) to test the hypothesis that one or more of these biomarkers were associated with $\mathrm{BPD} /$ mortality The following 14 proteins found in urine were considered: (i) albumin, (ii) $\beta$-2-microglobulin (B2M), (iii) cystatin C, (iv) Epidermal Growth Factor (EGF), (v) neutrophil gelatinase-associated lipocalin (NGAL), (vi) osteopontin (OPN), (vii) Uromodulin (UMOD), (viii) clusterin, (ix) kidney injury molecule 1 (KIM) $1,(\mathbf{x})$ osteoactivin, (xi) trefoil factor 3 (TFF3), (xii) vascular endothelia growth factor (VEGF), (xiii) calbindin, (xiv) alpha-glutathione S-transferase $(\alpha$-GST). We evaluated the associations between biomarkers and BPD/mortality outcomes controlling for possible demographic and intervention confounders, including gestational age, birth weight, "small for gestational age" (SGA), level of respiratory support during early days of life, and demographic information such as race and sex (8).

\section{METHODS \\ Study Population}

The study population included 113 premature infants admitted to the regional NICU at the University of Alabama at Birmingham (UAB) from February 2012 to June 2013. Families were approached for this study if infants met inclusion criteria. Inclusion criteria included a birth weight less than or equal to $1,200 \mathrm{~g}$, and/or a gestational age less than or equal to $31 \mathrm{wk}$. Infants were excluded if they suffered from a known congenital abnormality of the kidney or urinary tract. Enrolled infants were followed from the time of birth until $36 \mathrm{wk}$ PMA or hospital discharge, whichever happened first. The study was approved by the Institutional Review Board at UAB and all clinical 


\section{Articles Balena-Borreman e tal.}

and research practices were in accordance with the principles contained in the Declaration of Helsinki. Once informed parental consent was obtained, eligible infants were enrolled.

Infant and maternal demographics were collected after enrollment in the study. Race was self-reported as black, white, or other. Gestational age was recorded in completed weeks and days of gestation, and birth weight was measured in grams. The highest level of respiratory support was also recorded for postnatal days 1, 2, 3, and 4 . There were no differences in mean birth weight, gestational age, and 5 min Apgar score between infants enrolled in the study and those other eligible infants for whom consent was declined (Figure 1). Diagnosis of BPD at $36 \mathrm{wk}$ PMA was determined by the need for oxygen support at $36 \mathrm{wk}$ PMA, and no room air challenge was performed.

\section{Evaluation of Urine Biomarkers}

Urine was collected daily during the first $4 \mathrm{~d}$ of life using cuddle buns diapers (Small Beginnings, Hesperia, CA) placed at the perineum. Most infants had samples for the first $4 \mathrm{~d}$ of life (53\%), while $32 \%$ had three samples, $12 \%$ had two samples, and $4 \%$ had only one sample. The moist part of the diaper was cut out and placed in a syringe. Urine was squeezed into a centrifuge tube using the syringe plunger and centrifuged for $10 \mathrm{~min}$. The supernatant was aliquoted and stored frozen at $-70{ }^{\circ} \mathrm{C}$ until evaluation. Urine biomarker analysis was performed by electrochemiluminescence using multi-array plates assessed on a Meso Scale Discovery (MSD) Sector Imager 2400 (MSD, Gaithersburg, MD). Albumin, B2M, cystatin C, EGF, NGAL, OPN, and UMOD were assessed in urine samples using MSD Human Kidney Injury Panel 5 Kit assay, while $\alpha$ GST, calbindin, clusterin, KIM-1, osteoactivin, TFF3, and VEGF were measured using the MSD Human Kidney Injury Panel 3 Kit assay. Samples for the panel 3 assay were diluted 10-fold before being added to the plate. Samples for the panel 5 assays were diluted 500 -fold before being added to the plate. Samples were processed as indicated in the manufacturer's protocol and analyzed in the MSD Sector Imager. All biomarkers detected in the samples were within the range of the standard curve. Interrun percentage coefficient of variance $(\% \mathrm{CV})$ and intra-run $\% \mathrm{CV}$, the variability of samples and standards (between as well as in runs)

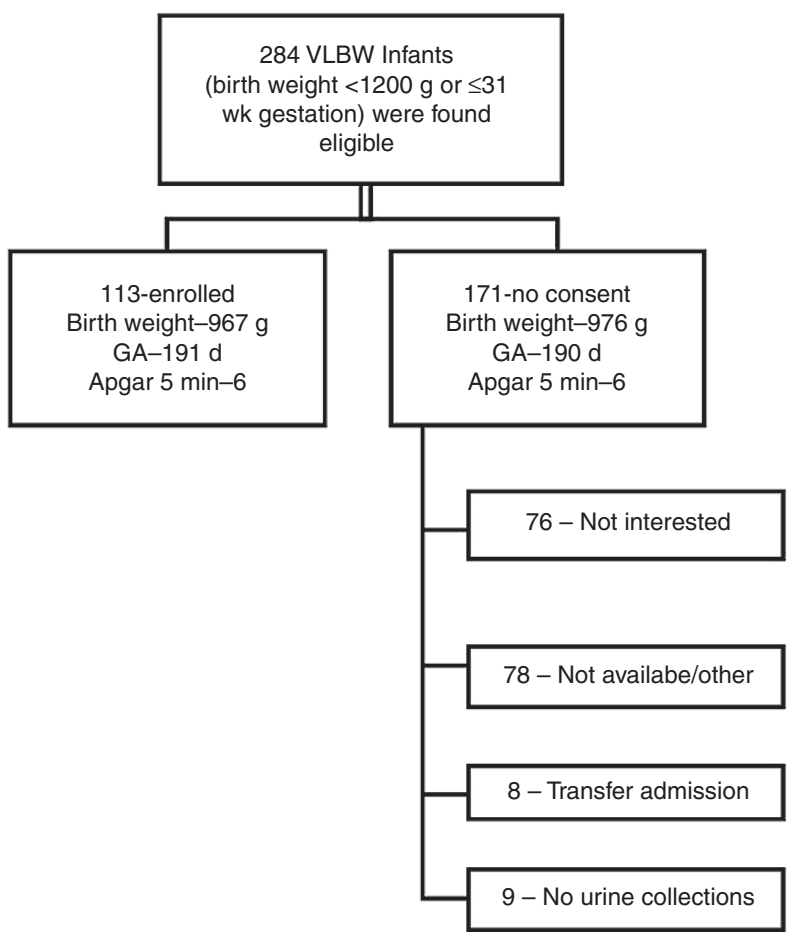

Figure 1. Breakdown enrolled and non-enrolled infants that met eligibility criteria for the study. Cr, creatinine; GA, gestational age; VLBW, very low birth weight. was below $10 \%$ in all cases, and below $5 \%$ in most sample/standard comparisons.

\section{Statistical Analysis}

The Shapiro-Wilk test and normal probability plots were used to assess the normality of all continuous variables. Descriptive statistics were found to determine differences between infants with and without the $\mathrm{BPD} /$ mortality outcome. $P<0.05$ was considered to be statistically significant. Normally distributed continuous variables were assessed using the Student's $t$-test, and categorical variables were compared using the chi-square test or Fisher's exact test when appropriate.

Measurements of raw concentrations of each biomarker were originally recorded in $\mathrm{pg} / \mathrm{ml}$ and those measurements below detection were recorded as $0.5 \mathrm{pg} / \mathrm{ml}$. All urine biomarker measurements were adjusted for urine creatinine to account for dilution differences in the urine samples. The adjusted urine biomarker measurements were then transformed by taking the natural logarithm $(\log )$ to normalize the distribution of the measurements.

We decided to use the maximum urine biomarker values measured in the first $4 \mathrm{~d}$ of life since higher levels of urine biomarker typically indicate peak injury. Evaluation of the minimum values for each biomarker, as well as assessment of the average values of the repeated biomarker measurements in the first $4 \mathrm{~d}$ of life provided similar results (data not shown). Therefore, we focused on the maximum adjusted biomarker values measured for each biomarker across days 1-4, disregarding within person variance of urine biomarker values measured, which is known to occur by day of life for some biomarkers (7).

A logistic regression model was used to provide unadjusted OR estimates of $\mathrm{BPD} /$ mortality for each biomarker (Table 3 ). In addition, we evaluated the associations of $\mathrm{BPD} /$ mortality with race, gender, birth weight, gestational age, and intubation status, as these demographics have previously been shown to be associated with BPD/mortality (8). Since gestational age and birth weight are highly correlated confounders, we chose to only include gestational age in the multivariable model. The additional contribution of birth weight to the $\mathrm{BPD} /$ mortality outcome, apart from gestational age, was accounted for by the inclusion of whether each infant was SGA. The multivariable model was chosen by stepwise selection with the entry criteria as $P<0.1$, and the removal criteria as $P>0.15$. Each model considered by stepwise selection was also assessed in light of the model likelihood ratio test. The final logistic regression model was chosen taking into account the stepwise selection results and the model likelihood ratio test. This final model provides OR estimates of $\mathrm{BPD} /$ mortality for selected biomarkers, while also controlling for known and measurable confounding variables.

Four additional indicators of model fit were also evaluated: area under the curve (AUC), R-Squared, Likelihood Ratio Model $P$-value, and Akaike information criterion. The R-Squared value ranges from 0 to 1 and provides the amount of explained variance. The Akaike information criterion statistic is useful to compare models, with lower values indicating better models. Finally, the AUC statistic is a joint measure of the specificity and sensitivity of a model, in this way an AUC of 0.5 predicts with the accuracy of a "coin flip", while an AUC = 1.0 provides $100 \%$ predictability.

\section{RESULTS}

Infants with $\mathrm{BPD} /$ mortality at $36 \mathrm{wk}$ PMA had significantly lower birth weight $(690 \pm 130$ vs. $1,115 \pm 130$ g; $P<0.0001)$, lower gestational age $(26 \pm 2$ vs. $29 \pm 1 \mathrm{wk} ; P<0.0001)$, were more likely to be intubated within the first $4 \mathrm{~d}$ of life (36 (88\%) vs. 19 (26\%); $P<0.0001)$, and they were more frequently SGA (10 (24\%) vs. $8(11 \%) ; P=0.06)$ (Table 1$)$.

Urine biomarker concentrations were divided by urine creatinine to account for differences in the dilution of samples. Differences in the values of the 14 urine biomarkers/cr over the first $4 \mathrm{~d}$ of life between those with and those without BPD are shown in Supplementary Table S1 online and Supplementary Figures S1-S7 online. Supplementary Table S1 online and 
Table 1. Demographic differences between infants with and without BPD/Mortality at 36 wk PMA

\begin{tabular}{|c|c|c|c|}
\hline $\begin{array}{l}\text { Infant } \\
\text { demographics }\end{array}$ & $\begin{array}{l}\text { BPD/mortality } \\
(N=41)\end{array}$ & $\begin{array}{c}\text { No BPD/ } \\
\text { mortality }(N=72)\end{array}$ & $P$ value \\
\hline $\begin{array}{l}\text { Gestational age } \\
\text { (weeks) }\end{array}$ & $26 \pm 2$ & $29 \pm 1$ & $<0.0001$ \\
\hline Birth weight (g) & $690 \pm 130$ & $1,115 \pm 130$ & $<0.0001$ \\
\hline Apgar 1 min & $2 \pm 1$ & $6 \pm 1$ & $<0.0001$ \\
\hline Apgar 5 min & $7 \pm 1$ & $8 \pm 0$ & $<0.0001$ \\
\hline $\begin{array}{l}\text { Small for gestational } \\
\text { age }\end{array}$ & $10(24 \%)$ & $8(11 \%)$ & 0.1 \\
\hline Gender (female) & $22(54 \%)$ & $36(50 \%)$ & 0.7 \\
\hline Race & & & 0.8 \\
\hline Black & $23(56 \%)$ & $37(51 \%)$ & \\
\hline White & $15(37 \%)$ & 31 (43\%) & \\
\hline Other & $3(7 \%)$ & $4(6 \%)$ & \\
\hline Intubation days $1-4$ & $36(88 \%)$ & $19(26 \%)$ & $<0.0001$ \\
\hline $\begin{array}{l}\text { Maternal } \\
\text { demographics }\end{array}$ & $\begin{array}{l}\text { BPD/mortality } \\
(N=41)\end{array}$ & $\begin{array}{c}\text { No BPD/ } \\
\text { mortality }(N=72)\end{array}$ & $P$ value \\
\hline Pre-eclampsia & $16(39 \%)$ & $17(24 \%)$ & 0.1 \\
\hline History of drug abuse & $1(2 \%)$ & $5(7 \%)$ & 0.4 \\
\hline $\begin{array}{l}\text { Antenatal } \\
\text { indomethacin }\end{array}$ & $5(12 \%)$ & $8(11 \%)$ & $>0.9$ \\
\hline Prenatal care & 40 (98\%) & $66(92 \%)$ & 0.4 \\
\hline Smoking & $3(7 \%)$ & $11(15 \%)$ & 0.2 \\
\hline Antenatal steroids & $40(98 \%)$ & $70(97 \%)$ & $>0.9$ \\
\hline Multiple births & $15(37 \%)$ & $21(29 \%)$ & 0.4 \\
\hline Chorioamnionitis & $18(44 \%)$ & $32(44 \%)$ & $>0.9$ \\
\hline Diabetes & $4(10 \%)$ & $8(11 \%)$ & $>0.9$ \\
\hline Hypertension & $9(22 \%)$ & $24(33 \%)$ & 0.2 \\
\hline \multicolumn{4}{|c|}{$\begin{array}{l}\text { Data are presented as median } \pm \text { SE or as frequency with percentage in parentheses. } \\
\text { The } P \text { value for all variables were estimated using the chi-square or Fisher's test for } \\
\text { categorical variables or the Wilcoxon Rank Sum test for continuous variables. } \\
\text { BPD, Bronchopulmonary dysplasia; PMA, postmenstrual age. }\end{array}$} \\
\hline
\end{tabular}

Supplementary Figures S1-S7 online illustrate how urine biomarker values can vary over time, and more importantly for the purpose of this study, how many of the biomarkers vary in those with and without $\mathrm{BPD} /$ mortality.

The remaining statistical analysis focused on the maximum biomarker levels in the first $4 \mathrm{~d}$ of life. To allow for modeling under normally distributed conditions, the values were adjusted by the natural logarithm $(\ln )$. The maximum biomarker levels in the first $4 \mathrm{~d}$ of life for $\beta$-2-microglobulin (B2M), cystatin $\mathrm{C}$, neutrophil gelatinase-associated lipocalin (NGAL), osteopontin (OPN), clusterin, kidney injury molecule 1 (KIM)1, trefoil factor 3 (TFF3), VEGF, and $\alpha$-glutathione S-transferase ( $\alpha$-GST) were significantly higher for those with $\mathrm{BPD} /$ mortality (Table 2 ). Table 3 provides the crude association between these demographic factors and these biomarkers with $\mathrm{BPD} /$ mortality. Of all the urine biomarkers measured, clusterin had the strongest association (odds ratio $(\mathrm{OR})=4.9$, 95\% CI: 2.5-9.5, $P<0.0001)$ with BPD/mortality. In addition,
Table 2. Differences in maximum Ln urine biomarker/creatinine $(\mathrm{Cr})$ between infants with and without BPD/mortality at 36 wk PMA

\begin{tabular}{|c|c|c|c|}
\hline $\begin{array}{l}\text { Maximum } \\
\text { biomarker levels } \\
\text { (days } 1 \text { to 4) }\end{array}$ & $\begin{array}{c}\text { BPD/mortality } \\
(N=41)\end{array}$ & $\begin{array}{c}\text { No BPD/ } \\
\text { mortality }(N=71)\end{array}$ & $P$ value \\
\hline Ln Albumin/Cr & $15.3(1.2)$ & $15.0(1.1)$ & 0.1 \\
\hline $\mathrm{LnB} 2 \mathrm{M} / \mathrm{Cr}$ & $14.1(0.8)$ & $13.8(0.6)$ & 0.01 \\
\hline Ln CystatinC/Cr & $12.1(0.9)$ & $10.7(1.2)$ & $<0.0001$ \\
\hline LnEGF/Cr & $4.6(0.5)$ & $4.8(0.3)$ & 0.01 \\
\hline Ln NGAL/Cr & $11.1(1.0)$ & $10.2(1.3)$ & 0.0003 \\
\hline $\mathrm{LnOPN} / \mathrm{Cr}$ & $11.5(0.9)$ & $10.7(0.6)$ & $<0.0001$ \\
\hline $\mathrm{Ln} \mathrm{UMOD/Cr}$ & $11.6(0.6)$ & $11.9(0.5)$ & 0.01 \\
\hline Ln Clusterin/Cr & $10.6(0.8)$ & $9.7(0.7)$ & $<0.0001$ \\
\hline Ln KIM1/Cr & $6.0(1.1)$ & $5.5(0.8)$ & 0.02 \\
\hline Ln Osteoactivin/Cr & $5.1(0.6)$ & $5.1(0.5)$ & 0.5 \\
\hline LnTFF3/Cr & $7.3(0.8)$ & $6.7(0.8)$ & $<0.0001$ \\
\hline Ln VEGF/Cr & $5.0(0.5)$ & $4.7(0.6)$ & 0.002 \\
\hline Ln Calbindin/Cr & $6.6(0.7)$ & $6.7(0.7)$ & 0.4 \\
\hline $\operatorname{Ln} \alpha-G S T / C r$ & $5.0(2.0)$ & $2.8(2.2)$ & $<0.0001$ \\
\hline
\end{tabular}

10 other urine biomarkers were associated with $\mathrm{BPD} /$ mortality (Table 3). Significant differences in many of these biomarkers are likely explained by the fact that the most premature infants have the most immature tubular function, allowing for loss of these proteins.

In order to prevent potential colinearity issues between biomarkers during modeling procedures, correlations between clusterin and the remaining biomarkers were considered, while also controlling for gestational age. We found that all correlations were lower than 0.8 , and therefore, collinearity problems between clusterin and other biomarkers were not anticipated in modeling (Table 4).

Because preterm infants have underdeveloped kidneys, which result in higher levels of protein in their urine, it is necessary to account for the degree of prematurity in order to make proper inferences. This is why gestational age was included in every multivariable model. After correcting for known confounders (GA, SGA, and intubation status), only urine clusterin and urine albumin were independently associated with $\mathrm{BPD} /$ mortality (Table 5 ).

Table 5 shows the sequential multivariable logistic regression models whereby we started with clinical data (GA and SGA) in model 1 , added intubation in model 2 , added clusterin in model 3 , and added albumin in model 4 . We then assessed whether including a third urine biomarker could significantly improve our model. However, none of the other biomarkers added substantially to the model. According to the R-squared statistic GA and SGA explained $29.6 \%$ of the variance, while intubation accounted for an additional $8.7 \%$. When clusterin was added to the model, it contributed an additional $9.2 \%$, and albumin explained an additional 3.4\%. The final model (which included GA, SGA, intubation, clusterin, and albumin) 
Table 3. Unadjusted logistic regression models for outcome BPD/ mortality at 36 wk PMA

\begin{tabular}{|c|c|c|}
\hline Demographic variables & OR (Cl) & $P$ value \\
\hline Gestational age & $0.6(0.5,0.7)$ & $<0.0001$ \\
\hline Birth weight & $0.6(0.5,0.8)$ & $<0.0001$ \\
\hline Small for gestational age (yes vs. no) & $2.6(0.9,7.4)$ & 0.1 \\
\hline Gender (F vs. M) & $1.9(0.6,2.6)$ & 0.7 \\
\hline Race & & 0.7 \\
\hline Black vs. White & $1.3(0.6,3.0)$ & \\
\hline Other vs. White & $1.6(0.3,7.9)$ & \\
\hline Intubation & OR (Cl) & $P$ value \\
\hline Intubation days 1-4 & & $<0.0001$ \\
\hline Yes vs. no & $19.7(6.7,57.6)$ & \\
\hline $\begin{array}{l}\text { Maximum biomarker levels } \\
\text { (days } 1 \text { to } 4 \text { ) }\end{array}$ & OR (Cl) & $P$ value \\
\hline Ln Albumin/Cr & $1.3(0.9,1.8)$ & 0.1 \\
\hline $\mathrm{Ln} B 2 \mathrm{M} / \mathrm{Cr}$ & $2.6(1.3,5.1)$ & 0.007 \\
\hline Ln CystatinC/Cr & $2.9(1.9,4.7)$ & $<0.0001$ \\
\hline $\mathrm{LnEGF/Cr}$ & $0.2(0.07,0.6)$ & 0.004 \\
\hline Ln NGAL/Cr & $1.9(1.3,2.8)$ & 0.0009 \\
\hline Ln OPN/Cr & $4.2(2.2,8.0)$ & $<0.0001$ \\
\hline Ln UMOD/Cr & $0.3(0.1,0.7)$ & 0.006 \\
\hline Ln Clusterin/Cr & $4.9(2.5,9.5)$ & $<0.0001$ \\
\hline Ln KIM1/Cr & $1.7(1.1,2.7)$ & 0.02 \\
\hline Ln Osteoactivin/Cr & $1.3(0.6,2.7)$ & 0.5 \\
\hline $\mathrm{LnTFF} 3 / \mathrm{Cr}$ & $3.2(1.7,5.9)$ & 0.0002 \\
\hline Ln VEGF/Cr & $2.8(1.4,5.6)$ & 0.005 \\
\hline Ln Calbindin/Cr & $0.8(0.5,1.4)$ & 0.4 \\
\hline $\operatorname{Ln} \alpha \mathrm{GST} / \mathrm{Cr}$ & $1.8(1.4,2.3)$ & $<0.0001$ \\
\hline
\end{tabular}

BPD, Bronchopulmonary dysplasia; PMA, postmenstrual age.

explained $50.9 \%$ of the variance in the $\mathrm{BPD} /$ mortality outcome. Clinical variables alone predicted $\mathrm{BPD} /$ mortality with an AUC of 0.884 . The addition of biomarkers clusterin and albumin to the model had an AUC of 0.941. After adjusting for GA, SGA, intubation and albumin, the odds of BPD/mortality by $\ln$ clusterin/cr was significant.

Intubation status in the first $4 \mathrm{~d}$ of life was included in the multivariable logistic regression model (Table 5) as a known confounding variable. However, intubation status could also be seen as an effect modifier rather than a mediator. For this reason, we report two different models in Table 6. The first model includes a group of 55 infants who were intubated at any time during the first $4 \mathrm{~d}$ of life-from which 36 infants died or developed BPD at $36 \mathrm{wk}$ PM. The second model includes a group of 57 infants-who were not intubated at any time during their first $4 \mathrm{~d}$ of life-from which only five infants died or developed BPD at $36 \mathrm{wk}$ PMA. In both models, clusterin was significantly associated with $\mathrm{BPD} /$ mortality. As expected, the prevalence of $\mathrm{BPD} /$ mortality was notably lower in those infants that were not intubated, however, the association
Table 4: Biomarker correlations with clusterin controlling for gestational age

\begin{tabular}{lcc}
\hline $\begin{array}{l}\text { Maximum } \\
\text { biomarker levels } \\
\text { (days 1 to 4) }\end{array}$ & $\begin{array}{c}\text { Correlation with maximum Ln } \\
\text { Clusterin/Cr level for days 1-4 } \\
\text { (controlling for gestational age) }\end{array}$ & P value \\
\hline Ln Albumin/Cr & 0.4 & $<0.0001$ \\
Ln B2M/Cr & 0.5 & $<0.0001$ \\
Ln CystatinC/Cr & 0.7 & $<0.0001$ \\
Ln EGF/Cr & 0.01 & 0.95 \\
Ln NGAL/Cr & 0.4 & $<0.0001$ \\
Ln OPN/Cr & 0.6 & $<0.0001$ \\
Ln UMOD/Cr & 0.01 & 0.9 \\
Ln KIM1/Cr & 0.3 & 0.002 \\
Ln Osteoactivin/Cr & 0.3 & 0.001 \\
Ln TFF3/Cr & 0.5 & $<0.0001$ \\
Ln VEGF/Cr & 0.5 & $<0.0001$ \\
Ln Calbindin/Cr & 0.3 & 0.007 \\
Ln $\alpha$ GST/Cr & 0.5 & $<0.0001$ \\
\hline
\end{tabular}

between clusterin and BPD was found to be higher in nonintubated infants (see also Table 6).

\section{DISCUSSION}

Using an available sample of clinical and urine biomarker data obtained in the first days of life, we examined the association between 14 urine biomarkers and $\mathrm{BPD} /$ mortality. After controlling for GA, SGA, and intubation status, urine clusterin and albumin were independently associated with BPD mortality. These associations were present in both intubated and nonintubated infants. The addition of these biomarkers to GA, SGA, and intubation status, improved $\mathrm{BPD} /$ mortality prediction models by an additional $13 \%$. These associations and the substantial model improvement suggest that urine biomarkers may improve the ability to predict $\mathrm{BPD} /$ mortality. Why these biomarker proteins are present in the urine, and whether these biomarkers may help identify infants at risk for $\mathrm{BPD} /$ mortality early in the disease course should be investigated.

While 12 of the 14 urine biomarkers tested were indeed associated with $\mathrm{BPD} /$ mortality, only two were independently associated with the composite outcome after accounting for important known confounders (GA, SGA, and intubation status). This is likely due to the fact that lower GA is associated with both $\mathrm{BPD} /$ mortality and higher urine proteins concentrations. Immature tubular function is the mostly probable explanation for the finding that urine proteins are highest in the most immature infants. Thus, inferences about urine biomarkers in premature infants must be made in the context of controlling for gestational age as a potential confounder.

In 2011, Laughon et al. (8) created predictive multivariable models for BPD at six postnatal ages: Day $1,3,7,14,21$, and 28. For each of these models, the same factors were included: (i) gestational age, (ii) birth weight, (iii) sex, (iv) race and ethnicity, (v) respiratory support, and (vi) fraction of inspired 
Table 5. Multivariable logistic regression models

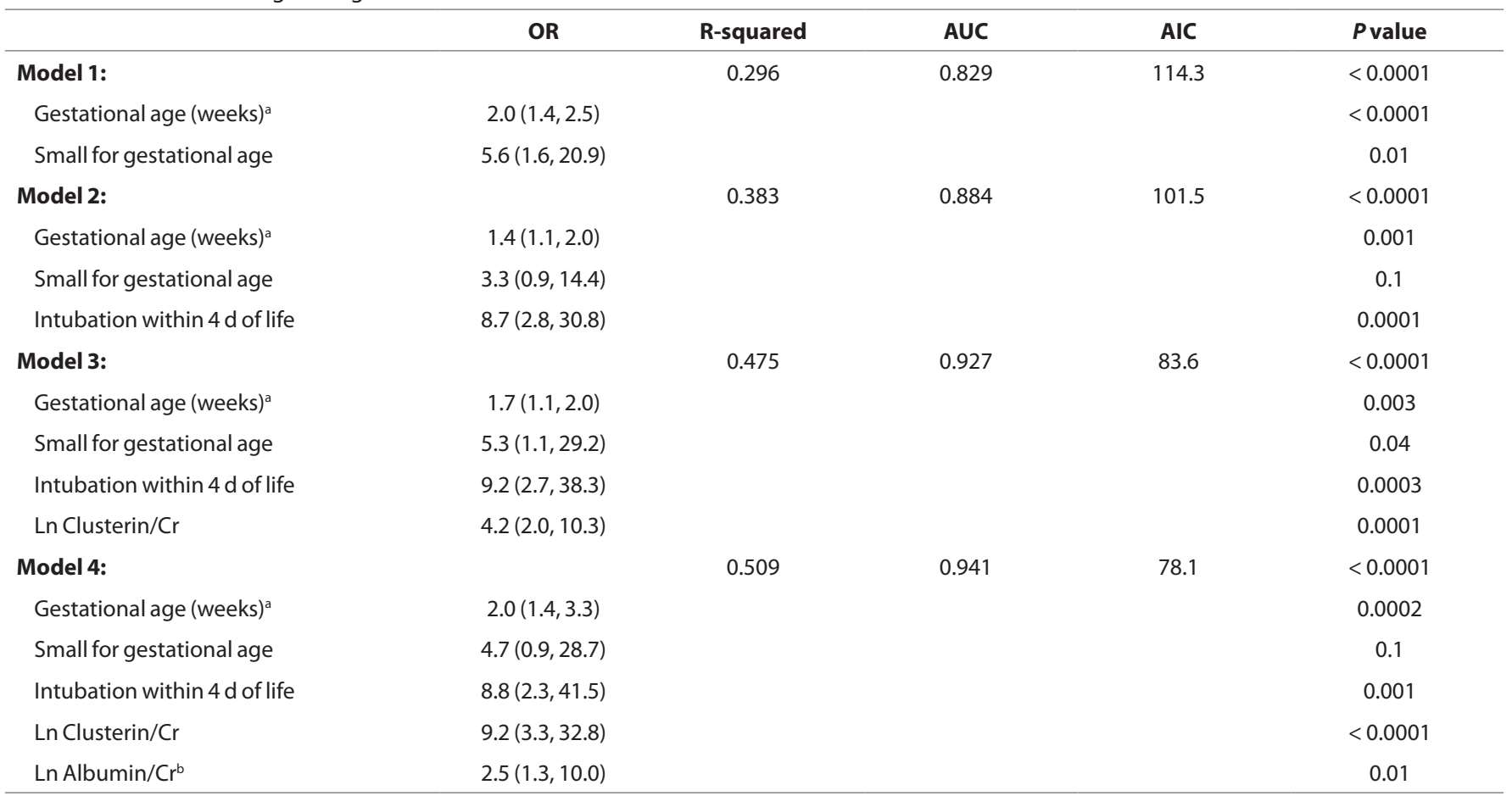

¿Lower GA is associated with higher risk of BPD/mortality. ' Lower max urine albumin associated with higher risk of BPD/mortality.

AIC, Akaike information criterion; AUC, area under the curve; BPD, Bronchopulmonary dysplasia; GA, gestational age.

Table 6. Multivariable logistic regression models stratified by intubation status

\begin{tabular}{|c|c|c|}
\hline Intubated $(N=55)$ & $\begin{array}{l}\text { BPD/mortality } \\
(N=36)\end{array}$ & \\
\hline$A U C=0.810$ & $A I C=60.9$ & Model $P=0.002$ \\
\hline R-squared = 0.275 & OR (Cl) & $P$ value \\
\hline Gestational age $^{a}$ & $1.7(1.1,2.5)$ & 0.003 \\
\hline Small for gestational age & $2.3(0.4,0.9)$ & 0.4 \\
\hline Ln Albumin/Cr & $2(0.8,5)$ & 0.1 \\
\hline Ln Clusterin/Cr & $4.8(1.5,15.6)$ & 0.003 \\
\hline Not intubated $(N=57)$ & BPD/mortality $(N=5)$ & \\
\hline AUC $=0.989$ & $A I C=19.2$ & Model $P<0.0001$ \\
\hline R-squared = 0.348 & OR (Cl) & $P$ value \\
\hline Gestational age $^{a}$ & $2.5(0.98,>10.0)$ & 0.1 \\
\hline Small for gestational age & $93.7(0.8,>999.99)$ & 0.1 \\
\hline Ln Albumin/Crb & $10.0(1.7,>1000)$ & 0.003 \\
\hline Ln Clusterin/Cr & $316.6(7.7,>999.99)$ & $<0.0001$ \\
\hline
\end{tabular}

aLower GA is associated with higher risk of BPD/mortality. 'Lower max urine albumin associated with higher risk of BPD/mortality.

AIC, Akaike information criterion; AUC, area under the curve; BPD, Bronchopulmonary dysplasia.

oxygen. They found that GA and degree of respiratory support were the most predictive factors for BPD. The multivariable models, which include all six clinical factors noted above, had the following C Statistics (comparable to AUC): day $1=0.793$, day $3=0.807$, day $7=0.818$, day $14=0.827$, day $21=0.836$, and day $28=0.854$. In comparison, our models-which included both clinical and biomarker data from days 1 to $4-$ had an AUC statistic of 0.941 .

In 2013, Onland et al. (9) reviewed 26 clinical prediction models for BPD based on the factors present in the first week of life and concluded that the model created by Ryan in 1998 as well as the one proposed by Laughon in 2011 (8) were the best models for BPD. The internally derived AUC statistics of these models were 0.85 and 0.81 , respectively. Our internally derived AUC statistic based only on clinical factors was 0.884 , while our internally derived AUC statistic increased to 0.941 when the two biomarkers were also included in the model.

Since intubation was such a significant contributor to BPD, we felt it was important to stratify our cohort into intubated and nonintubated infants. We also felt that evidence showing that clusterin was associated with BPD in both intubated and nonintubated infants would strengthen our hypothesis that this was a plausible association. There are several possible explanations on why the association between clusterin and BPD is higher in nonintubated infants. First, because the baseline risk for BPD is higher for intubated vs. nonintubated infants (intubation is the biggest risk factor for BPD in our study), urine biomarkers may have a lower impact on who develops BPD in this higher risk group. Second, statistical imprecision-due to small sample size-could also contribute to the higher OR.

Interestingly, the association between urine albumin/cr proved to reverse in direction when going from the unadjusted to the adjusted model (a.k.a. "Simpson's Paradox"). Although the unadjusted logistic regression model in Table 3 suggests 


\section{Articles | Balena-Borneman et al.}

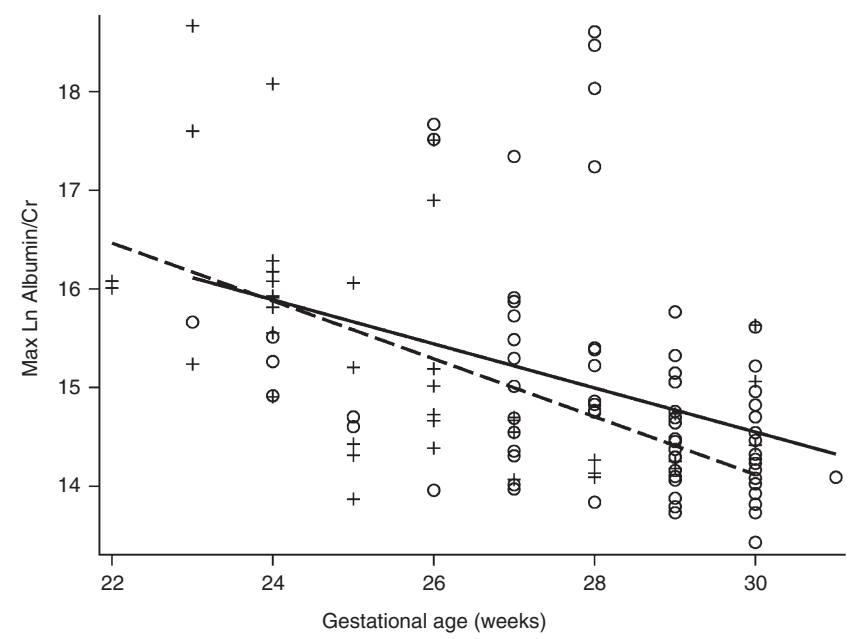

Figure 2. Maximum adjusted albumin levels for postnatal days 1 to 4 across gestational age by BPD/mortality status. Key: BPD/mortality status at 36 wk PMA. No trend line ——. No data points: $\bigotimes$. Yes trend line: - - - - - -. Yes data points: +. BPD, ; Cr, creatinine; PMA, postmenstrual age.

that higher levels of albumin are associated with $\mathrm{BPD} /$ mortality, this is reversed in the adjusted model (Table 5). The likely explanation for this finding is covariable selection, in this case, gestational age. As shown in Figure 2, lower levels of albumin are actually associated with BPD/Mortality after controlling for gestational age. The more significant driving force behind the unadjusted association between elevated urine albumin and $\mathrm{BPD} /$ mortality was the decrease in urine albumin levels with the increase in gestational age. This known relationship between gestational age and urine albumin lead to the reversal of the direction in the adjusted models. This finding was confirmed by the multivariable logistic regression model (Table 5).

One strength of our study is that the data was obtained from a recent and relatively large, prospective cohort of very preterm infants. Our sensitivity analysis suggested no differences between those enrolled and those not enrolled, allowing for generalization to our NICU. We were able to show that regardless of whether an infant was intubated or not, urine biomarkers, and particularly clusterin, can help stratify patients early in their life as predictors of significant clinical outcomes. Despite these strengths, we also acknowledge a few limitations. One of these limitations is that not all infants had urine samples collected for all $4 \mathrm{~d}$; however, it is unlikely that individuals with fewer than four urine samples introduced systematic bias, since there was no apparent discrimination in the number of samples between those with and those without BPD/mortality. Second, as evidenced by our models, not all variability can be explained by the variables and confounding factors that were included in the analysis, and therefore, it is possible that other modifying effects exist. Third, while the sample size was sufficient to investigate the association between maximum adjusted level of each biomarker in the first $4 \mathrm{~d}$ of life and BPD/ mortality, it was not sufficient to model the trend of the biomarkers levels over time, or to determine whether the average value over time was better than the maximum value. A larger cohort would be needed to understand how changes in urine clusterin could predict clinical cutoffs. Our sample size, lack of a validation cohort, and the study design limit our ability to provide clinically significant cut points, make inferences as to the pathophysiology of these biomarkers, or allow for clinical utility at this time.

The primary biomarker found to be associated with $\mathrm{BPD} /$ mortality was clusterin. Clusterin, also known as Apolipoprotein J, is a $75 \mathrm{kDA}$ disulfide-linked heterodimeric protein and it is ubiquitously expressed. Functionally, this is a secreted glycoprotein that has a central role in cellular processes such as lipid transport, membrane recycling, protein folding, cell lysis, and apoptosis (10-13). Interestingly, clusterin is an unusual chaperon that is associated with protein folding of secreted proteins in the Golgi apparatus (13), and has emerged as a biosensor of oxidative injury that has been shown to improve stress resistance in fruit flies (14). Clusterin has been linked to many physiological processes and disorders (15). The association between clusterin and BPD is certainly plausible given that clusterin levels have been linked to lung function, particularly in the case of asthma (16). In the kidney, this protein is found mainly in the cytoplasm of the renal distal and collecting tubular cells (17). Although our study was not designed to determine the pathophysiological mechanisms linking clusterin and BPD, several possible hypotheses may explain our findings. First, elevated clusterin levels may signal kidney damage and clusterin may play a role in the repair process after AKI. In vitro models have shown that clusterin protects renal tubular cells from aminoglycoside toxicity (18), and since the association between AKI and lung injury has been described in animals and humans (19-22), perhaps elevated urine clusterin could be a marker of this process. Since we recently showed an independent association between AKI and $\mathrm{BPD}$ in premature infants (5), it is possible that urine proteins associated with $\mathrm{BPD} /$ mortality represent the negative effects of $\mathrm{AKI}$ on $\mathrm{BPD} /$ mortality. A second possibility is that clusterin is upregulated in the lung in those with BPD. Elevated levels of clusterin have been found in severe asthma in the lung, and they were inversely correlated with pulmonary function (16). Elevated clusterin in asthma may have a beneficial effect by reducing CCL20-mediated dendritic cell recruitment, and therefore, reducing intracellular reactive oxygen species (23). High expression of lung clusterin may lead to high systemic levels, which would then be excreted in high concentrations in the kidney. This would suggest that urine clusterin is a surrogate for systemic and/or lung clusterin. A third possibility is that clusterin may be a marker of systemic oxidative stress and/ or a marker of severity of illness that can be detected in urine.

In conclusion, urine levels of clusterin and albumin are independently associated with $\mathrm{BPD} /$ mortality at $36 \mathrm{wk}$ PMA and improve prediction models. Although we cannot advocate for clinical utility of these potential biomarkers at this time, validation of these associations in other cohorts is warranted. If these associations persist in validation cohorts, studies to determine how these biomarkers may be combined with clinical data will be needed, understanding the optimal time-points and cutoffs 
would be necessary, and further understanding of the pathophysiology of these relationships would be also needed.

\section{SUPPLEMENTARY MATERIAL}

Supplementary material is linked to the online version of the paper at http:// www.nature.com/pr

\section{ACKNOWLEDGMENTS}

The authors wish to thank Emma Perez-Costas, PhD (Department of Pediatrics, University of Alabama at Birmingham) who performed substantive science editing as well as proofreading of this manuscript.

\section{STATEMENT OF FINANCIAL SUPPORT}

The research reported in this publication was supported by a Norman Siegel Career Development Award from the American Society of Nephrology, the University of Alabama at Birmingham (UAB) O'Brien Center, and Grant NIH P30-DK079337. D.A. receives funding from NIH (R01 DK13608-01) and the Pediatric and Infant Center for Acute Nephrology (PICAN), which is sponsored by Children's of Alabama and UAB's School of Medicine, as well as by the Department of Pediatrics, and the Center for Clinical and Translational Science (CCTS; award \#UL1TR00165). N.A. receives funding from NIH (grants \# U01 HL122626; R01 HD067126; R01 HD066982; U10 HD34216). J.B-B. received funding from the National Heart, Lung, and Blood Institute (grant \# T32HL079888 (PI: Tiwari)). H.K.T. receives funding from UAB Department of Biostatistics. R.L.G. receives funding from UAB CCTS and PICAN.

Disclosure: D.A. is on the speaker bureau for Baxter Renal Products-Baxter International (Deerfield, Illinois, USA), Acute Kidney Injury Foundation (Cincinnati Children's Hospital, Cincinnati, Ohio, USA), and BTG pharmaceuticals-BTG International Inc. (West Conshohocken, Pennsylvania, USA).

\section{REFERENCES}

1. Ambalavanan N, Carlo WA. Bronchopulmonary dysplasia: new insights. Clin Perinatol 2004;31:613-28.

2. Coalson JJ. Pathology of bronchopulmonary dysplasia. Semin Perinatol 2006;30:179-84.

3. Tooley WH. Epidemiology of bronchopulmonary dysplasia. J Pediatr 1979;95(5 Pt 2):851-8.

4. Shennan AT, Dunn MS, Ohlsson A, Lennox K, Hoskins EM. Abnormal pulmonary outcomes in premature infants: prediction from oxygen requirement in the neonatal period. Pediatrics 1988;82:527-32.

5. Askenazi D, Patil NR, Ambalavanan N, et al. Acute kidney injury is associated with bronchopulmonary dysplasia/mortality in premature infants. Pediatr Nephrol 2015;30:1511-8.

6. Askenazi DJ, Koralkar R, Patil N, et al. Acute kidney injury urine biomarkers in very low-birth-weight infants. Clin J Am Soc Nephrol 2016;1: 1527-35.
7. Saeidi B, Koralkar R, Griffin RL, Halloran B, Ambalavanan N, Askenazi DJ. Impact of gestational age, sex, and postnatal age on urine biomarkers in premature neonates. Pediatr Nephrol 2015;30:2037-44.

8. Laughon MM, Langer JC, Bose CL, et al.; Eunice Kennedy Shriver National Institute of Child Health and Human Development Neonatal Research Network. Prediction of bronchopulmonary dysplasia by postnatal age in extremely premature infants. Am J Respir Crit Care Med 2011;183: 1715-22.

9. Onland W, Debray TP, Laughon MM, et al. Clinical prediction models for bronchopulmonary dysplasia: a systematic review and external validation study. BMC Pediatr 2013;13:207.

10. Jones SE, Jomary C. Clusterin. Int J Biochem Cell Biol 2002;34:427-31.

11. Koltai T. Clusterin: a key player in cancer chemoresistance and its inhibition. Onco Targets Ther 2014;7:447-56.

12. Sansanwal P, Li L, Sarwal MM. Inhibition of intracellular clusterin attenuates cell death in nephropathic cystinosis. J Am Soc Nephrol 2015;26: $612-25$.

13. Lin CC, Tsai P, Sun HY, et al. Apolipoprotein J, a glucose-upregulated molecular chaperone, stabilizes core and NS5A to promote infectious hepatitis C virus virion production. J Hepatol 2014;61:984-93.

14. Lee YN, Shim YJ, Kang BH, Park JJ, Min BH. Over-expression of human clusterin increases stress resistance and extends lifespan in Drosophila melanogaster. Biochem Biophys Res Commun 2012;420:851-6.

15. Trougakos IP. The molecular chaperone apolipoprotein J/clusterin as a sensor of oxidative stress: implications in therapeutic approaches - a minireview. Gerontology 2013;59:514-23.

16. Li JY, Liu J, Jiang J, et al. Calcium oxalate calculi-induced clusterin expression in kidney. Urolithiasis 2015;43:411-8.

17. Girton RA, Sundin DP, Rosenberg ME. Clusterin protects renal tubular epithelial cells from gentamicin-mediated cytotoxicity. Am J Physiol Renal Physiol 2002;282:F703-9.

18. Kwon HS, Kim TB, Lee YS, et al. Clusterin expression level correlates with increased oxidative stress in asthmatics. Ann Allergy Asthma Immunol 2014;112:217-21.

19. Doi K, Ishizu T, Fujita T, Noiri E. Lung injury following acute kidney injury: kidney-lung crosstalk. Clin Exp Nephrol 2011;15:464-70.

20. Ko GJ, Rabb H, Hassoun HT. Kidney-lung crosstalk in the critically ill patient. Blood Purif 2009;28:75-83.

21. Karimi Z, Ketabchi F, Alebrahimdehkordi N, et al. Renal ischemia/reperfusion against nephrectomy for induction of acute lung injury in rats. Ren Fail. 2016;3:1-16.

22. Faubel S, Shah PB. Immediate consequences of acute kidney injury: the impact of traditional and nontraditional complications on mortality in acute kidney injury. Adv Chronic Kidney Dis 2016;23:179-85.

23. Hong GH, Kwon HS, Moon KA, et al. Clusterin modulates allergic airway inflammation by attenuating CCL20-mediated dendritic cell recruitment. J Immunol 2016;196:2021-30. 